\title{
Raumwissenschaften als interdisziplinäres Forschungsfeld
}

\author{
Andreas Klee • Gregor Prinzensing
}

Online publiziert: 27. September 2011

(C) Springer-Verlag 2011

Raumforschung und Raumordnung versteht sich als interdisziplinär konzipierte wissenschaftliche Zeitschrift. Vereinfacht gesagt stehen der Raum und seine Nutzung im Vordergrund der Diskussionen. Aus verschiedenen Disziplinen werden gesellschaftlich relevante raumwirksame Prozesse beschrieben, erklärt und bewertet. Daraus entsteht ein Kaleidoskop theoretischer, methodischer und anwendungsbezogener Auseinandersetzungen mit dem Raum, den in ihm stattfindenden Prozessen und der auf ihn projizierten Vorstellungen. Auch in diesem Heft befassen sich Autorinnen und Autoren aus unterschiedlichen Blickwinkeln und disziplinären Verortungen mit diesem Themenfeld.

Der Geograph Olaf Kühne wagt in seinem Beitrag Heimat und sozial nachhaltige Landschaftsentwicklung eine Zusammenschau der Begriffe, Konzepte und Vorstellungen von Heimat, Landschaft und Nachhaltigkeit. Dabei nimmt er eine dezidiert konstruktivistische Perspektive ein: Heimat und Landschaft sind demnach konstitutiv sozial konstruiert. Er integriert Heimat als soziales Bedürfnis in das Konzept nachhaltiger Entwicklung. Infolgedessen lässt sich der Umgang mit Landschaft - verkürzt dargestellt - nicht länger nur als Umgang mit ihren physischen Dimensionen verstehen. Vielmehr, so lautet eine Erkenntnis Kühnes, erlangen die sozialen und individuellen Deutungen von Landschaft und die Ansprüche der ortsansässigen Bevölkerung an Vorstellungen von Heimat eine vermehrte Bedeutung. Plane-

Dr. A. Klee $(\bowtie)$

Akademie für Raumforschung und Landesplanung -,

Leibniz-Forum für Raumwissenschaften, Hohenzollernstraße 11,

30161 Hannover, Deutschland

E-Mail: Klee@ARL-net.de

G. Prinzensing

Leibniz-Institut für Regionalentwicklung und Strukturplanung,

Flakenstraße 28-31, 15537 Erkner, Deutschland

E-Mail: prinzensing@irs-net.de rinnen und Planer müssen demnach im Sinne einer sozial nachhaltigen Entwicklung akzeptieren, dass mehrere Diskurse über Landschaft existieren: sowohl Laien- als auch Expertendiskurse. Kühne gelingt somit nicht nur eine Verknüpfung von subjektiv erfahrbarer Heimat, sozial konstruierter Landschaft und planerischer Nachhaltigkeitsdiskurse, sondern auch von raumbezogenen Diskursen in Sozialwissenschaften, räumlicher Planung und Ökologie.

Eine besonders interessante Verknüpfung von Ökonomie und räumlicher Planung nehmen Kati Volgmann und Wolfgang Knapp in ihrem Beitrag Neue ökonomische Kerne in nordrhein-westfälischen Stadtregionen: Postsuburbanisierung und Restrukturierung kernstädtischer Räume vor. Der Beitrag geht von jüngeren Entwicklungen in Stadtregionen aus: postsuburbane Entwicklungen am Rand der Regionen und gleichzeitige Revitalisierungen von Kernstädten. Dabei wird der Fokus auf die Entwicklung neuer Beschäftigtenund Unternehmenskonzentrationen außerhalb der alten Kerne gelegt. Volgmann und Knapp stellen in ihrem Beitrag heraus, dass in den untersuchten Stadtregionen in Nordrhein-Westfalen diskontinuierliche, räumlich differenzierte Entwicklungsdynamiken zu finden sind. Sowohl wieder stärker gewordene Innenstädte als auch ,neue ökonomische Kerne" an den Rändern haben spezielle ökonomische Funktionen. Sie sind untereinander verflochten. Manche der untersuchten suburbanen gewerblichen Standorte haben ganz eigene funktionale Profile entwickelt und stehen in weltweiten Austauschbeziehungen und nicht - wie man häufig denken könnte - in einem unmittelbaren Abhängigkeitsverhältnis von der jeweiligen Kernstadt.

Der Ökonom Christoph Vietze widmet sich einer verkehrspolitisch und -planerisch bedeutsamen Frage. In seinem Beitrag Preiselastizitäten der Nachfrage im ÖPNV unter spezieller Berücksichtigung ermäßigter Sozialtarife am Beispiel einer mittelgroßen Stadt untersucht er mögli- 
che Nachfrageänderungen nach Mobilitätsdienstleistungen in Abhängigkeit der Einführung ermäßigter Tarife. Als Beispiel dient die Stadt Jena, in der ermäßigte Sozialtarife für bestimmte Bevölkerungsgruppen eingeführt wurden. Vietze konnte mit seiner Untersuchung andere Studien dieser Art im Wesentlichen bestätigen, wonach kleine und ländlich strukturierte Städte eine vergleichsweise hohe Preiselastizität aufweisen. Die Ergebnisse, die mit ökonometrischen Verfahren ermittelt wurden, sind für die räumliche Planung, insbesondere für die Verkehrsplanung, sehr wichtig. Denn die Informationen werden benötigt, um den Zuschussbedarf der Kommunen an den ÖPNV zu ermitteln, der erforderlich wird, wenn Sozialtarife für benachteiligte Bevölkerungsgruppen eingeführt werden.

Der vierte Beitrag dieses Heftes kommt aus der Feder von Erik Gawel und Stefan Möckel und trägt den Titel Zur regionalen Differenzierung von Wassernutzungsabgaben. Hintergrund sind die in Deutschland unterschiedlich geregelten Wassernutzungsabgaben in Form der bundesweit einheitlichen Abwasserabgabe und der länderspezifischen Wasser- entnahmeentgelte. Sie werden zwar von Land zu Land in unterschiedlicher Weise erhoben, jedoch jeweils ohne regionalen Knappheitsbezug - und dies bei unterschiedlichen gewässerökologischen und -morphologischen Bedingungen. Grund genug für die beiden Autoren, Gawel ist Volkswirt, Möckel Jurist, sich mit den Möglichkeiten und Grenzen regionalisierter Wassernutzungsabgaben in Deutschland zu befassen. Der Schwerpunkt ihrer Untersuchung liegt in ökonomischen und juristischen Gesichtspunkten. Sie kommen zum Schluss, dass ökologische, ökonomische und rechtliche Gründe für eine bundesweit einheitlich konzipierte Wassernutzungsabgabe sprechen, die jedoch in unterschiedlicher Höhe regionenspezifisch vorgesehen werden kann. Kriterien für die Staffelungshöhe könnten demnach der vorfindliche Wasserkörper und der Verursacherbeitrag der einzelnen Wassernutzer sein.

Wie immer, hoffen wir, mit diesen vielschichtigen Beiträgen das Interesse unserer Leserinnen und Leser wecken zu können und wünschen viele neue Anregungen und Erkenntnisse. 